\title{
Cardiovascular disease behavioral risk factors among Latinos by citizenship and documentation status
}

\author{
Alexander N. Ortega ${ }^{1 *} \mathbb{D}$, Jessie Kemmick Pintor ${ }^{1}$, Brent A. Langellier ${ }^{1}$, Arturo Vargas Bustamante ${ }^{2}$, \\ Maria-Elena De Trinidad Young ${ }^{3}$, Michael L. Prelip², Cinthya K. Alberto ${ }^{1}$ and Steven P. Wallace ${ }^{2}$
}

\begin{abstract}
Background: Studies have observed that recent Latino immigrants tend to have a physical health advantage compared to immigrants who have been in the US for many years or Latinos who are born in the United States. An explanation of this phenomenon is that recent immigrants have positive health behaviors that protect them from chronic disease risk. This study aims to determine if trends in positive cardiovascular disease (CVD) risk behaviors extend to Latino immigrants in California according to citizenship and documentation status.

Methods: We examined CVD behavioral risk factors by citizenship/documentation statuses among Latinos and non-Latino US-born whites in the 2011-2015 waves of the California Health Interview Survey. Adjusted multivariable logistic regressions estimated the odds for CVD behavioral risk factors, and analyses were stratified by sex.

Results: In adjusted analyses, using US-born Latinos as the reference group, undocumented Latino immigrants had the lowest odds of current smoking, binge drinking, and frequency of fast food consumption. There were no differences across the groups for fruit/vegetable intake and walking for leisure. Among those with high blood pressure, undocumented immigrants were least likely to be on medication. Undocumented immigrant women had better patterns of CVD behavioral risk factors on some measures compared with other Latino citizenship and documentation groups.

Conclusions: This study observes that the healthy Latino immigrant advantage seems to apply to undocumented female immigrants, but it does not necessarily extend to undocumented male immigrants who had similar behavioral risk profiles to US-born Latinos.
\end{abstract}

Keywords: Immigrants, Hispanic Americans, Citizenship, Cardiovascular disease risk factors, Cardiovascular disease prevention, Heart disease

\section{Background}

Cardiovascular disease (CVD) accounts for 20\% of deaths among Latinos [1]. Latinos have disparities in clinical (e.g., blood pressure, BMI) and behavioral (e.g., fast food and soda consumption, fruits/vegetable intake, exercising, and blood pressure medication use) risk

\footnotetext{
* Correspondence: ano37@drexel.edu

'Dornsife School of Public Health, Drexel University, Philadelphia, PA 19104, USA

Full list of author information is available at the end of the article
}

factors for CVD [2, 3], although there is significant within group variability [4, 5]. Research has observed variations among Latinos in CVD risk factors and outcomes based on factors such as sex, nativity, language use, time in the United States (US) and generational status $[4,5]$. One factor that has not been well studied in the CVD and Latino health literature is citizenship and documentation status and its association with CVD risk factors and outcomes. Citizenship and documentation status (i.e., undocumented, documented, naturalized

C C The Author(s). 2020 Open Access This article is licensed under a Creative Commons Attribution 4.0 International License, which permits use, sharing, adaptation, distribution and reproduction in any medium or format, as long as you give appropriate credit to the original author(s) and the source, provide a link to the Creative Commons licence, and indicate if changes were made. The images or other third party material in this article are included in the article's Creative Commons licence, unless indicated otherwise in a credit line to the material. If material is not included in the article's Creative Commons licence and your intended use is not permitted by statutory regulation or exceeds the permitted use, you will need to obtain permission directly from the copyright holder. To view a copy of this licence, visit http://creativecommons.org/licenses/by/4.0/. The Creative Commons Public Domain Dedication waiver (http://creativecommons.org/publicdomain/zero/1.0/) applies to the data made available in this article, unless otherwise stated in a credit line to the data. 
citizen) influence immigrants' migration and integration trajectories in the US [6], which may potentially influence the behavioral and environmental factors that increase the risk of CVD. Documentation status is particularly salient since the majority (66\%) of undocumented immigrants have been in the US for more than 10 years [7], and as they get older their risk for CVD will increase.

Latino immigrant documentation status is associated with access to and utilization of health care, with studies showing undocumented Latino immigrants having lower rates of insurance, usual sources of care and use of health services than other US-born or immigrant Latinos [8-11] Having access to primary care has been shown to be positively associated with CVD behavioral prevention among Latinos [12]. Using a Los Angeles-based survey that included in-person blood pressure measurements, Young and Pebley (2017) found that undocumented Latino immigrants had higher rates of high blood pressure than documented Latino immigrants, and they had similar rates of high blood pressure to those who were USborn [13]. Moreover, while documented immigrants who had been in the US for less than 15 years had lower rates of high blood pressure compared to their documented counterparts who had been in the country more than 15 years, they found that time in the US was not associated with blood pressure for undocumented immigrants [13]. These findings suggest that there was not a healthy immigrant effect for undocumented immigrants. A recent study using pooled 2011-2015 waves of the California Health Interview Survey (CHIS) found that while undocumented Latinos had the worst patterns of access to and use of health services, they had better self-reported CVD clinical risk profiles, such as lower rates of overweight/obesity, high blood pressure and diabetes compared with other Latino immigrants and those who were US-born [10].

To our knowledge, there has been no research on the associations among citizenship and documentation status and CVD behavioral risk factors among Latinos using a large statewide representative sample. Thus, this study examines whether self-reported CVD behavioral risk factors varied by citizenship and documentation statuses among Latinos in California. Using pooled 20112015 waves of CHIS, which is the largest state-level health and health care survey in the US and one of the only population-based, representative surveys with measures of immigrant documentation statuses, we examine the relationships among citizenship and documentation statuses (i.e., US-born, naturalized, green card holder, undocumented) and CVD behavioral risk factors (i.e., smoking, binge drinking, fast food and soda consumption, fruits/vegetable intake, walking, and blood pressure medication use) for nonelderly Latino adults.

\section{Methods}

\section{Study population and data source}

We use data from the 2011-2015 waves of the California Health Interview Survey (CHIS). CHIS is a representative, cross-sectional survey of the noninstitutionalized population in California. The survey is administered in five languages including Spanish and is conducted via a dual-frame random-digit-dial design (both landline and cellphone). Since 2011, CHIS has been an ongoing survey with data collected continuously during a two-year data collection cycle. We limit our sample to nonelderly (ages 18-64 years) Latino and US-born non-Latino white adults for a total sample of 51,386 participants. More details on CHIS are available elsewhere [14].

\section{Measures}

\section{Citizenship and documentation status}

CHIS is the only state-level population-based representative survey which measures both citizenship and documentation statuses for immigrants. CHIS asks participants about their race, Latino ethnicity and nativity. To determine citizenship status, CHIS asks participants who are foreign-born "are you a citizen of the United States?" Citizens are then asked, "when did you become naturalized?," while noncitizens are asked, "are you a permanent resident with a green card?" Following previous peer-reviewed studies [9-11, 15-17], immigrant participants who are noncitizens and do not have a green card are classified as undocumented. We use information on citizenship and documentation statuses to create the following mutually exclusive groups for Latinos: US-born (8303), naturalized (3879), green card (3369) undocumented (3053). We also include nonLatino US-born whites as a reference group $(32,782)$. A previous analysis of CHIS data found that using the "no green card" status as a proxy for "undocumented" and relying on self- reports for green card and naturalized citizenship status is valid and results in only a 5\% misclassification error [18].

\section{CVD behavioral risk factors}

We measured CVD behavioral risk factors through the following CHIS measures: current smoking status (current smoker, currently not smoking), having engaged in binge drinking in the past year (5 or more drinks for males on one occasion, 4 or more drinks for females on one occasion), having eaten fast food in the past week, the total number of times ate fast food in the past week, having drunk soda at least once in the past week, the total number of times drank soda in the past week, having eaten fruits or vegetables at least 5 times per day in the past week, the number of times the participant walked for $10 \mathrm{~min}$ or more for leisure in the past week, and, for those with a physician diagnosis of high blood 
pressure, if the participant was currently taking blood pressure medication. The "having eaten fruits or vegetables at least 5 times per day in the past week" measure was derived from a weekly measure. We summed the weekly consumption of fruits and vegetables and then divided by seven to obtain a daily measure. We chose five as the threshold since the United States Department of Agriculture recommends that adults should consume 1.5-2.0 cups of fruit and 2.0-3.0 cups of vegetables per day [19].

Independent Measures.

Our independent measures include sex (male, female), marital status (married, not married), age (18-34, 35-49, 50-64), English language proficiency (speaks only English, very well or well; speaks English not well or not at all), employment status (currently employed, not currently employed), income as a percentage of the federal poverty level (FPL) (0-138\%, 139-250\%, 251-400\%, $\geq 400 \%$ ), location of residence (urban, not urban), education (less than high school, high school, more than high school), insurance status (currently insured, currently uninsured), self-reported health status (poor, fair, good, very good, excellent) and survey year.

\section{Statistical analyses}

To account for the complex sampling design of CHIS and for non-response, we used survey weights and design variables in all analyses. First, we conducted chisquared analyses to assess variation across citizenship and documentation statuses and our independent measures. Second, we conducted chi-squared and analysis of variance (ANOVA) analyses to assess significant variations across citizenship and documentation statuses and our CVD behavioral risk factor measures. Given previously observed differences in CVD risk factors by sex among Latinos $[4,5]$, the second set of analyses were stratified by sex (male/female). Lastly, we ran multivariable regression analyses to assess the associations among citizenship and documentation status and CVD behavioral risk factors, adjusting for the independent variables. Binary outcomes were modeled using multivariable logistic regressions, and count outcomes were modeled using multivariable Poisson regression. We report odds ratios (ORs) and 95\% confidence intervals (CIs) for all logistic regressions and incidence rate ratios (IRRs) and 95\% CI's for Poisson regressions. Stata, version 14, was used for the statistical analyses.

\section{Results}

In Table 1, we showed the sample characteristics for Latinos by citizenship and documentation status and for non-Latino whites. Undocumented Latino immigrants had the highest proportion of any documentation status group of younger adults, the lowest proportion who speak English well or very well, the highest proportion under $138 \%$ of FPL, the lowest levels of education and lowest proportion with health insurance. The noncitizen groups were similar in having low proportions of respondents who reported excellent or very good health status.

In Table 2, we showed CVD behavioral risk factors stratified by sex for Latinos by citizenship and documentation status and for non-Latino whites.

\section{Smoking and binge drinking}

Undocumented Latino immigrants were overall less likely to be current smokers than all other groups except naturalized immigrants. There were no differences in current smoking by documentation status for men and, among women, undocumented immigrants were the least likely to currently smoke. Overall and among men, undocumented and naturalized Latino immigrants were the least likely to report binge drinking in the last year, and women holding green cards had the lowest proportion of those reporting binge drinking among women.

\section{Fast food, soda and fruit and vegetable intake}

Any fast food consumption in the past week was reported by a lower proportion of all immigrant groups than US-born Latinos, although at a higher proportion than US-born whites, with similar patterns for men and women. The number of times in the past week respondents ate fast food was lower among all groups of immigrant men than US-born Latino men and higher than US-born white men. Among women, however, undocumented immigrants had the lowest average frequency of eating fast food in the past week across all groups. Undocumented immigrants also had the highest proportion of having drank soda at least once in the past week and having drank soda the highest number of times in the past week; this was consistent for men and women. Undocumented immigrants had the lowest proportion reporting that they ate fruits and vegetables at least 5 times per day. While undocumented immigrant men were less likely to report eating fruits and vegetables at least 5 times per day than all other groups, undocumented women were more likely to have eaten fruits and vegetables than their US-born and naturalized counterparts.

\section{Leisure walking}

Overall, undocumented Latino immigrants and green card holders reported the lowest frequency of leisure walking of at least $10 \mathrm{~min}$ in the past week compared to the other groups. Across the immigrant groups, men had similar frequencies of leisure walking, and undocumented female immigrants had the lowest frequency of all groups. 
Table 1 Descriptive Statistics by Race/Ethnicity and Citizen and Documentation Status, California Residents Ages 18-64, 2011-2015

\begin{tabular}{|c|c|c|c|c|c|c|c|}
\hline & \multirow{2}{*}{$\begin{array}{l}\text { Total } \\
(N=51,386)\end{array}$} & \multicolumn{4}{|c|}{ Latino $(n=18,604)$} & \multirow{2}{*}{$\begin{array}{l}\text { Non-Latino } \\
\text { White } \\
\text { US-born } \\
(N=32,782)\end{array}$} & \multirow[t]{2}{*}{$p$-value ${ }^{a}$} \\
\hline & & $\begin{array}{l}\text { US-born } \\
(N=8303)\end{array}$ & $\begin{array}{l}\text { Naturalized Citizens } \\
(N=3879)\end{array}$ & $\begin{array}{l}\text { Green Card Holders } \\
(N=3369)\end{array}$ & $\begin{array}{l}\text { Undocumented Residents } \\
(N=3053)\end{array}$ & & \\
\hline Female & 49.70 & 49.28 & 53.91 & 49.40 & 48.84 & 49.29 & 0.0226 \\
\hline Married & 49.20 & 31.30 & 67.65 & 61.32 & 43.57 & 53.03 & $<0.001$ \\
\hline \multicolumn{8}{|l|}{ Age } \\
\hline $18-34$ & 37.76 & 63.05 & 17.21 & 25.21 & 37.07 & 32.13 & $<0.001$ \\
\hline $35-49$ & 32.43 & 22.36 & 40.36 & 45.39 & 52.48 & 29.33 & \\
\hline $50-64$ & 29.81 & 14.59 & 42.43 & 29.41 & 10.45 & 38.55 & \\
\hline $\begin{array}{l}\text { Speaks English Well } \\
\text { or Very Well }\end{array}$ & 81.20 & 97.99 & 57.52 & 30.58 & 16.85 & 99.94 & $<0.001$ \\
\hline Currently Employed & 72.22 & 69.65 & 72.51 & 71.16 & 69.13 & 74.22 & $<0.001$ \\
\hline \multicolumn{8}{|l|}{ FPL } \\
\hline $0-138$ & 28.81 & 29.69 & 35.71 & 54.64 & 71.99 & 13.67 & $<0.001$ \\
\hline $139-250$ & 18.21 & 22.38 & 27.55 & 27.61 & 19.14 & 12.42 & \\
\hline $251-400$ & 16.80 & 19.95 & 19.54 & 10.43 & 6.25 & 17.99 & \\
\hline 400 or above & 36.17 & 27.99 & 17.20 & 7.33 & 2.62 & 55.92 & \\
\hline Urban Area & 88.58 & 92.37 & 93.18 & 88.64 & 92.20 & 85.09 & $<0.001$ \\
\hline \multicolumn{8}{|l|}{ Education } \\
\hline Less Than High School & 19.30 & 9.04 & 39.46 & 58.15 & 64.63 & 4.06 & $<0.001$ \\
\hline High School & 25.03 & 35.61 & 26.57 & 21.06 & 22.99 & 20.73 & \\
\hline More than High School & 55.67 & 55.35 & 33.97 & 20.78 & 12.38 & 75.21 & \\
\hline Currently Insured & 80.13 & 79.91 & 78.62 & 68.21 & 46.84 & 89.36 & $<0.001$ \\
\hline \multicolumn{8}{|l|}{ Self-Reported Health } \\
\hline Excellent & 18.95 & 17.29 & 15.96 & 10.45 & 10.44 & 23.61 & $<0.001$ \\
\hline Very Good & 30.17 & 31.83 & 19.75 & 12.85 & 14.55 & 37.75 & \\
\hline Good & 31.11 & 32.65 & 36.00 & 38.66 & 39.36 & 26.35 & \\
\hline Fair & 16.26 & 14.20 & 24.00 & 33.77 & 32.30 & 9.30 & \\
\hline Poor & 3.51 & 4.04 & 4.29 & 4.27 & 3.36 & 2.99 & \\
\hline \multicolumn{8}{|l|}{ Survey Year } \\
\hline 2011 & 19.75 & 19.29 & 20.39 & 18.98 & 16.15 & 20.70 & 0.001 \\
\hline 2012 & 19.91 & 19.12 & 20.59 & 20.92 & 20.03 & 19.94 & \\
\hline 2013 & 20.03 & 21.26 & 18.18 & 19.22 & 20.52 & 19.86 & \\
\hline 2014 & 20.09 & 20.41 & 21.04 & 21.55 & 19.30 & 19.64 & \\
\hline 2015 & 20.22 & 19.91 & 19.80 & 19.33 & 24.00 & 19.86 & \\
\hline
\end{tabular}

Unless otherwise indicated data are expressed as column percentages

${ }^{a}$ Calculated by means of $X^{2}$ tests across Latino and US-born non-Latino white samples

\section{Blood pressure medication}

Among those with a physician diagnosis of high blood pressure, undocumented immigrants had much lower rates of currently taking blood pressure medication, and this disparity was even more pronounced for women.

\section{Adjusted multivariable regressions}

When controlling for the independent measures, undocumented immigrants had better CVD risk behaviors than US-born Latinos in three of eight categories (smoking, binge drinking, and number of times ate fast food) (Table 3). There were no categories where undocumented immigrants had worse risk profiles than US-born Latinos. In the stratified analyses, undocumented men had better behaviors only in number of times ate fast food, while undocumented women had lower odds of smoking, binge drinking, number of times ate fast food, and number of times having drank soda. Undocumented women who 
Table 2 CVD Behavioral Risk Factors by Race/Ethnicity and Citizenship and Documentation Status, California Residents Ages 18-64, 2011-2015

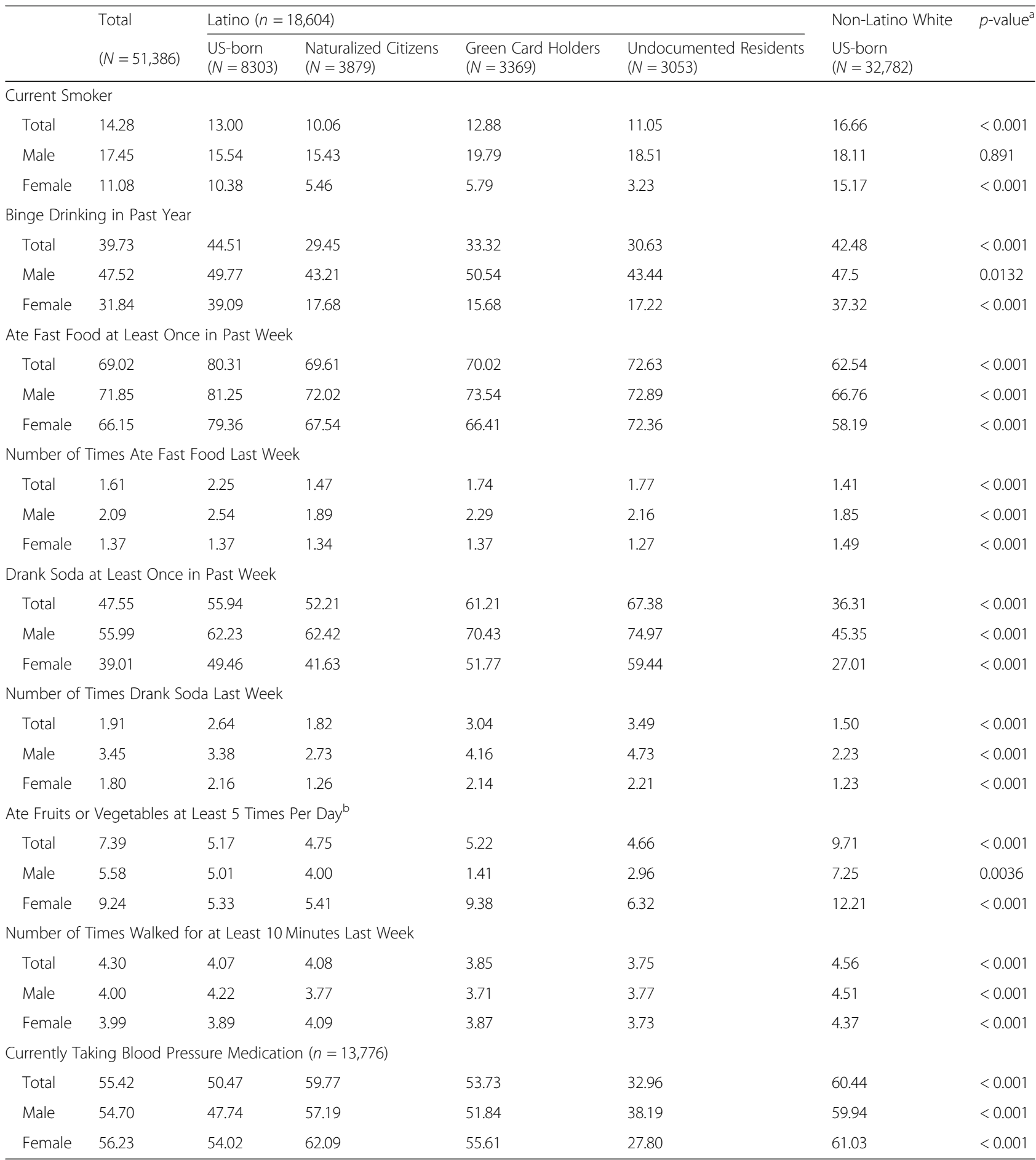

Unless otherwise indicated data are expressed as column percentages

${ }^{a}$ Calculated by means of $x^{2}$ and analysis of variance tests across Latino and US-born non-Latino white samples

${ }^{b}$ Derived from a weekly measure

reported hypertension had a substantially lower odds of taking blood pressure medication than US-born Latinos, which was the only negative comparison among all the stratified comparisons.
Compared to US-born Latinos, US-born non-Latino whites had higher odds of smoking and binge drinking and lower odds of eating any/more often fast food, drinking any/more often soda and higher odds 
Table 3 Multivariable Analyses of CVD Behavioral Risk Factors by Race/Ethnicity and Citizenship and Documentation Status, California Residents Ages 18-64, 2011-2015

\begin{tabular}{|c|c|c|c|c|c|c|}
\hline & \multirow[t]{2}{*}{ Model } & \multicolumn{4}{|c|}{ Latino $(n=18,604)$} & \multirow{2}{*}{$\begin{array}{l}\text { Non-Latino White } \\
\text { US-born }\end{array}$} \\
\hline & & US-born & Naturalized Citizens & Green Card Holders & Undocumented Residents & \\
\hline \multicolumn{7}{|c|}{ Current Smoker } \\
\hline All & $\begin{array}{l}\text { Logistic (OR) } \\
{[\mathrm{Cl}]}\end{array}$ & REF & $\begin{array}{l}0.82 \\
{[0.63,1.05]}\end{array}$ & $\begin{array}{l}0.90 \\
{[0.68,1.21]}\end{array}$ & $\begin{array}{l}0.61^{* *} \\
{[0.46,0.81]}\end{array}$ & $\begin{array}{l}2.02^{* * *} \\
{[1.74,2.35]}\end{array}$ \\
\hline Men & $\begin{array}{l}\text { Logistic (OR) } \\
{[\mathrm{Cl}]}\end{array}$ & REF & $\begin{array}{l}1.08 \\
{[0.77,1.52]}\end{array}$ & $\begin{array}{l}1.17 \\
{[0.82,1.68]}\end{array}$ & $\begin{array}{l}0.81 \\
{[0.57,1.16]}\end{array}$ & $\begin{array}{l}1.86^{* * *} \\
{[1.55,2.24]}\end{array}$ \\
\hline Women & $\begin{array}{l}\text { Logistic (OR) } \\
{[\mathrm{Cl}]}\end{array}$ & REF & $\begin{array}{l}0.55^{* *} \\
{[0.37,0.82]}\end{array}$ & $\begin{array}{l}0.58 \\
{[0.33,1.01]}\end{array}$ & $\begin{array}{l}0.27^{* * *} \\
{[0.16,0.47]}\end{array}$ & $\begin{array}{l}2.26^{* * *} \\
{[1.82,2.81]}\end{array}$ \\
\hline \multicolumn{7}{|c|}{ Binge Drinking in Past Year } \\
\hline All & $\begin{array}{l}\text { Logistic (OR) } \\
{[\mathrm{Cl}]}\end{array}$ & REF & $\begin{array}{l}0.86 \\
{[0.74,1.01]}\end{array}$ & $\begin{array}{l}0.99 \\
{[0.83,1.18]}\end{array}$ & $\begin{array}{l}0.75^{* *} \\
{[0.62,0.91]}\end{array}$ & $\begin{array}{l}1.15^{* *} \\
{[1.06,1.25]}\end{array}$ \\
\hline Men & $\begin{array}{l}\text { Logistic (OR) } \\
{[\mathrm{Cl}]}\end{array}$ & REF & $\begin{array}{l}1.15 \\
{[0.92,1.44]}\end{array}$ & $\begin{array}{l}1.42^{* *} \\
{[1.10,1.81]}\end{array}$ & $\begin{array}{l}0.87 \\
{[0.66,1.14]}\end{array}$ & $\begin{array}{l}1.15^{*} \\
{[1.01,1.30]}\end{array}$ \\
\hline Women & $\begin{array}{l}\text { Logistic (OR) } \\
{[\mathrm{Cl}]}\end{array}$ & REF & $\begin{array}{l}0.62^{* * *} \\
{[0.49,0.79]}\end{array}$ & $\begin{array}{l}0.60^{* * *} \\
{[0.45,0.78]}\end{array}$ & $\begin{array}{l}0.62^{* *} \\
{[0.45,0.85]}\end{array}$ & $\begin{array}{l}1.15^{* * *} \\
{[1.01,1.31]}\end{array}$ \\
\hline \multicolumn{7}{|c|}{ Fast Food at Least Once in Past Week } \\
\hline All & $\begin{array}{l}\text { Logistic (OR) } \\
{[\mathrm{Cl}]}\end{array}$ & REF & $\begin{array}{l}0.80^{*} \\
{[0.66,0.95]}\end{array}$ & $\begin{array}{l}0.81^{*} \\
{[0.66,1.00]}\end{array}$ & $\begin{array}{l}0.84 \\
{[0.67,1.05]}\end{array}$ & $\begin{array}{l}0.55^{* * *} \\
{[0.50,0.62]}\end{array}$ \\
\hline Men & $\begin{array}{l}\text { Logistic (OR) } \\
{[\mathrm{Cl}]}\end{array}$ & REF & $\begin{array}{l}0.84 \\
{[0.64,1.11]}\end{array}$ & $\begin{array}{l}0.92 \\
{[0.68,1.24]}\end{array}$ & $\begin{array}{l}0.83 \\
{[0.59,1.17]}\end{array}$ & $\begin{array}{l}0.61^{* * *} \\
{[0.52,0.72]}\end{array}$ \\
\hline Women & $\begin{array}{l}\text { Logistic (OR) } \\
{[\mathrm{Cl}]}\end{array}$ & REF & $\begin{array}{l}0.76^{*} \\
{[0.61,0.94]}\end{array}$ & $\begin{array}{l}0.71^{*} \\
{[0.53,0.96]}\end{array}$ & $\begin{array}{l}0.85 \\
{[0.61,1.17]}\end{array}$ & $\begin{array}{l}0.51^{* * *} \\
{[0.44,0.58]}\end{array}$ \\
\hline \multicolumn{7}{|c|}{ Number of Times Ate Fast Food Last Week } \\
\hline All & $\begin{array}{l}\text { Poisson (IRR) } \\
{[\mathrm{Cl}]}\end{array}$ & REF & $\begin{array}{l}0.82^{* * *} \\
{[0.76,0.90]}\end{array}$ & $\begin{array}{l}0.90^{*} \\
{[0.81,1.00]}\end{array}$ & $\begin{array}{l}0.82^{* * *} \\
{[0.73,0.91]}\end{array}$ & $\begin{array}{l}0.79^{* * *} \\
{[0.74,0.83]}\end{array}$ \\
\hline Men & $\begin{array}{l}\text { Poisson (IRR) } \\
{[\mathrm{Cl}]}\end{array}$ & REF & $\begin{array}{l}0.85^{* *} \\
{[0.76,0.95]}\end{array}$ & $\begin{array}{l}0.97 \\
{[0.85,1.11]}\end{array}$ & $\begin{array}{l}0.83^{*} \\
{[0.71,0.97]}\end{array}$ & $\begin{array}{l}0.85^{* * *} \\
{[0.79,0.92]}\end{array}$ \\
\hline Women & $\begin{array}{l}\text { Poisson (IRR) } \\
{[\mathrm{Cl}]}\end{array}$ & REF & $\begin{array}{l}0.79^{* * *} \\
{[0.71,0.88]}\end{array}$ & $\begin{array}{l}0.80^{* *} \\
{[0.70,0.93]}\end{array}$ & $\begin{array}{l}0.79^{* * *} \\
{[0.70,0.90]}\end{array}$ & $\begin{array}{l}0.71^{* * *} \\
{[0.65,0.77]}\end{array}$ \\
\hline \multicolumn{7}{|c|}{ Drank Soda at Least Once in Past Week } \\
\hline All & $\begin{array}{l}\text { Logistic (OR) } \\
{[\mathrm{Cl}]}\end{array}$ & REF & $\begin{array}{l}1.00 \\
{[0.85,1.17]}\end{array}$ & $\begin{array}{l}1.11 \\
{[0.93,1.33]}\end{array}$ & $\begin{array}{l}1.14 \\
{[0.93,1.39]}\end{array}$ & $\begin{array}{l}0.67^{* * *} \\
{[0.61,0.74]}\end{array}$ \\
\hline Men & $\begin{array}{l}\text { Logistic (OR) } \\
{[\mathrm{Cl}]}\end{array}$ & REF & $\begin{array}{l}1.17 \\
{[0.92,1.50]}\end{array}$ & $\begin{array}{l}1.29^{*} \\
{[1.00,1.66]}\end{array}$ & $\begin{array}{l}1.29 \\
{[0.96,1.73]}\end{array}$ & $\begin{array}{l}0.74^{* * *} \\
{[0.65,0.85]}\end{array}$ \\
\hline Women & $\begin{array}{l}\text { Logistic (OR) } \\
{[\mathrm{Cl}]}\end{array}$ & REF & $\begin{array}{l}0.87 \\
{[0.70,1.07]}\end{array}$ & $\begin{array}{l}0.96 \\
{[0.75,1.22]}\end{array}$ & $\begin{array}{l}0.98 \\
{[0.75,1.28]}\end{array}$ & $\begin{array}{l}0.61^{* * *} \\
{[0.53,0.69]}\end{array}$ \\
\hline \multicolumn{7}{|c|}{ Number of Times Drank Soda Last Week (Count - Poisson IRR) } \\
\hline All & $\begin{array}{l}\text { Poisson (IRR) } \\
{[\mathrm{Cl}]}\end{array}$ & REF & $\begin{array}{l}0.73^{* * *} \\
{[0.63,0.85]}\end{array}$ & $\begin{array}{l}0.93 \\
{[0.79,1.10]}\end{array}$ & $\begin{array}{l}0.85 \\
{[0.73,1.00]}\end{array}$ & $\begin{array}{l}0.89^{*} \\
{[0.81,0.99]}\end{array}$ \\
\hline Men & $\begin{array}{l}\text { Poisson (IRR) } \\
{[\mathrm{Cl}]}\end{array}$ & REF & $\begin{array}{l}0.80^{*} \\
{[0.66,0.96]}\end{array}$ & $\begin{array}{l}0.98 \\
{[0.79,1.22]}\end{array}$ & $\begin{array}{l}0.92 \\
{[0.74,1.13]}\end{array}$ & $\begin{array}{l}0.90 \\
{[0.80,1.02]}\end{array}$ \\
\hline Women & $\begin{array}{l}\text { Poisson (IRR) } \\
{[\mathrm{Cl}]}\end{array}$ & REF & $\begin{array}{l}0.63^{* * *} \\
{[0.50,0.80]}\end{array}$ & $\begin{array}{l}0.86 \\
{[0.68,1.09]}\end{array}$ & $\begin{array}{l}0.74^{*} \\
{[0.58,0.95]}\end{array}$ & $\begin{array}{l}0.89 \\
{[0.75,1.05]}\end{array}$ \\
\hline \multicolumn{7}{|c|}{ Ate Fruits or Vegetables at Least 5 Times Per Day ${ }^{a}$} \\
\hline All & $\begin{array}{l}\text { Logistic (OR) } \\
{[\mathrm{Cl}]}\end{array}$ & REF & $\begin{array}{l}0.89 \\
{[0.53,1.52]}\end{array}$ & $\begin{array}{l}1.04 \\
{[0.59,1.85]}\end{array}$ & $\begin{array}{l}1.01 \\
{[0.48,2.15]}\end{array}$ & $\begin{array}{l}1.73^{* * *} \\
{[1.28,2.33]}\end{array}$ \\
\hline Men & $\begin{array}{l}\text { Logistic (OR) } \\
{[\mathrm{Cl}]}\end{array}$ & REF & $\begin{array}{l}0.75 \\
{[0.29,1.92]}\end{array}$ & $\begin{array}{l}0.24^{* *} \\
{[0.09,0.63]}\end{array}$ & $\begin{array}{l}0.50 \\
{[0.12,1.98]}\end{array}$ & $\begin{array}{l}1.51 \\
{[0.96,2.37]}\end{array}$ \\
\hline Women & $\begin{array}{l}\text { Logistic (OR) } \\
{[\mathrm{Cl}]}\end{array}$ & REF & $\begin{array}{l}1.06 \\
{[0.57,1.97]}\end{array}$ & $\begin{array}{l}2.10^{*} \\
{[1.08,4.10]}\end{array}$ & $\begin{array}{l}1.67 \\
{[0.73,3.82]}\end{array}$ & $\begin{array}{l}1.93^{* *} \\
{[1.31,2.84]}\end{array}$ \\
\hline
\end{tabular}


Table 3 Multivariable Analyses of CVD Behavioral Risk Factors by Race/Ethnicity and Citizenship and Documentation Status, California Residents Ages 18-64, 2011-2015 (Continued)

\begin{tabular}{|c|c|c|c|c|c|c|}
\hline & \multirow[t]{2}{*}{ Model } & \multicolumn{4}{|c|}{ Latino $(n=18,604)$} & \multirow{2}{*}{$\begin{array}{l}\text { Non-Latino White } \\
\text { US-born }\end{array}$} \\
\hline & & US-born & Naturalized Citizens & Green Card Holders & Undocumented Residents & \\
\hline \multicolumn{7}{|c|}{ Number of Times Walked for at Least 10 Minutes Last Week (Count - Poisson IRR) } \\
\hline All & $\begin{array}{l}\text { Poisson (IRR) } \\
{[\mathrm{Cl}]}\end{array}$ & REF & $\begin{array}{l}0.98 \\
{[0.89,1.08]}\end{array}$ & $\begin{array}{l}0.97 \\
{[0.89,1.04]}\end{array}$ & $\begin{array}{l}0.96 \\
{[0.88,1.04]}\end{array}$ & $\begin{array}{l}1.07^{*} \\
{[1.01,1.13]}\end{array}$ \\
\hline Men & $\begin{array}{l}\text { Poisson (IRR) } \\
{[\mathrm{Cl}]}\end{array}$ & REF & $\begin{array}{l}0.91 \\
{[0.83,1.00]}\end{array}$ & $\begin{array}{l}0.93 \\
{[0.84,1.04]}\end{array}$ & $\begin{array}{l}0.95 \\
{[0.86,1.05]}\end{array}$ & $\begin{array}{l}1.05 \\
{[0.97,1.14]}\end{array}$ \\
\hline Women & $\begin{array}{l}\text { Poisson (IRR) } \\
{[\mathrm{Cl}]}\end{array}$ & REF & $\begin{array}{l}1.04 \\
{[0.91,1.20]}\end{array}$ & $\begin{array}{l}1.00 \\
{[0.91,1.12]}\end{array}$ & $\begin{array}{l}0.98 \\
{[0.87,1.11]}\end{array}$ & $\begin{array}{l}1.08 \\
{[1.00,1.16]}\end{array}$ \\
\hline \multicolumn{7}{|c|}{ Currently Taking Blood Pressure Medication $(n=13,776)$} \\
\hline All & $\begin{array}{l}\text { Logistic (OR) } \\
{[\mathrm{Cl}]}\end{array}$ & REF & $\begin{array}{l}0.73 \\
{[0.52,1.02]}\end{array}$ & $\begin{array}{l}0.83 \\
{[0.53,1.29]}\end{array}$ & $\begin{array}{l}0.59^{*} \\
{[0.36,0.95]}\end{array}$ & $\begin{array}{l}0.83 \\
{[0.67,1.03]}\end{array}$ \\
\hline Men & $\begin{array}{l}\text { Logistic (OR) } \\
{[\mathrm{Cl}]}\end{array}$ & REF & $\begin{array}{l}0.66 \\
{[0.40,1.08]}\end{array}$ & $\begin{array}{l}0.82 \\
{[0.41,1.658]}\end{array}$ & $\begin{array}{l}0.99 \\
{[0.49,2.00]}\end{array}$ & $\begin{array}{l}0.80 \\
{[0.60,1.06]}\end{array}$ \\
\hline Women & $\begin{array}{l}\text { Logistic (OR) } \\
{[\mathrm{Cl}]}\end{array}$ & REF & $\begin{array}{l}0.76 \\
{[0.47,1.23]}\end{array}$ & $\begin{array}{l}0.82 \\
{[0.46,1.48]}\end{array}$ & $\begin{array}{l}0.35^{* *} \\
{[0.18,0.67]}\end{array}$ & $\begin{array}{l}0.87 \\
{[0.63,1.20]}\end{array}$ \\
\hline
\end{tabular}

Adjusted for sex, marital status, health insurance, age, education, English language proficiency, employment, federal poverty level, urban/rural area, self-reported health, and survey year

${ }^{a}$ Derived from a weekly measure

$\mathrm{Cl}$ denotes a 95\% confidence interval; OR denotes an odds ratio; IRR denotes an incidence rate ratio

${ }^{* * *} p<0.001,{ }^{* *} p<0.01,{ }^{*} p<0.05$

of fruit/vegetable consumption and leisure walking (Table 3).

\section{Discussion}

To our knowledge, this is the first study to examine the relationships among citizenship and documentation statuses and CVD behavioral risk factors for Latinos using a large, state-level representative survey. Our findings suggest that the behavioral risk factors of undocumented Latino immigrants are sometimes better and rarely worse when compared with US-born Latinos, while nonLatino whites are sometimes better and sometimes worse than US-born Latinos. The most important contribution of this study is showing that most of the favorable risk factor trends among undocumented residents are driven by the better odds for women but not for men, with the exception of hypertension treatment. These findings are consistent with those of Young and Pebley (2017), who observed that there was not a healthy immigrant effect for high blood pressure for undocumented Latino immigrants [13].

The demographic profile of undocumented Latino immigrants has changed over the past three decades. In $1995,33 \%$ of undocumented immigrants had been in the US for more than ten years, and in 2016 that percent rose to $66 \%$ [7]. Also, recent Latino immigrants are more likely to come from urban areas in Latin America and from Central America than in the past [20]. In the US, over $70 \%$ of undocumented immigrants are Latino, and $51 \%$ of undocumented Latino immigrants come from Mexico [21, 22]; this percent is even higher in California, where $69 \%$ of undocumented immigrants are from Mexico [21]. In our study, while not significant in the adjusted analysis, undocumented Latino immigrants were the most likely to report drinking soda in the past week in the unadjusted analysis; this unadjusted finding might reflect longstanding consumption patterns in Mexico, where sugar-sweetened beverage consumption is high [23] and contributes $69 \%$ of added sugars in the Mexican diet [24]. Sweetened beverage consumption in Mexico, as well as the high rate of obesity $(33 \%$ in Mexico compared to $40 \%$ in the US) [25], prompted the country to pass a one-peso-per-liter tax that has resulted in a decrease in consumption by up to $10 \%$ [26]. This decreased consumption among the Mexican population may not "spill over" to Latino immigrants in the US, because most immigrants have lived in the US for more than a decade, and migration from Mexico into the US is low relative to the size of the existing immigrant population from Central America [7, 27]. Of course, this does not explain why green card holders have the lowest proportion reporting having drank soda. The relationship between diet and documentation status is likely complex and involves multiple levels of influence including living in enclaves where access to healthy foods is poor $[28,29]$.

The frequency of fast food consumption in the past week was lower for all immigrants versus US-born Latinos. This is likely due to the low level of consumption of food prepared away from home in Mexico and other Latin American countries [30]. Consumption and spending on food prepared away from home are particularly 
low among Mexicans with demographic characteristics similar to those of migrants, particularly those from rural areas and with low levels of income and education. In fact, the positive relationship between socioeconomic status and food prepared away from home among the Mexican population mirrors our findings of higher fast food consumption among US-born Latinos than among immigrants. We did not find any differences across the groups for fruit and vegetable consumption.

Undocumented Latinos were the only immigrant group that had lower odds of current smoking and binge drinking compared with US-born Latinos. This is consistent with the findings of a claims-based study in Southern California that found low rates of smoking and drinking among undocumented immigrants in a county health system [31]. While we did not find differences in leisure walking, it should be noted that this measure does not account for other forms of physical activity such as work-related physical activity (e.g., farming, construction) or transportation.

A recent paper showed that the Patient Protection and Affordable Care Act (ACA) reduced hypertension treatment disparities for Mexican-heritage Latinos. That study, however, did not look at the effects among Latino immigrant groups according to citizenship or documentation status [32]. Our findings show that undocumented immigrants who self-reported a physician diagnosis of high blood pressure were the least likely to be taking blood pressure medication compared with US-born Latinos, even after adjusting for health insurance.

Other studies have found that CVD risk profiles for Latinos vary by sex $[4,5]$. In our study, we found that sex moderated some associations between citizenship and documentation statuses and CVD behavioral risk factors, such as current smoking and binge drinking, where undocumented immigrant Latinas had lower rates than US-born Latinas. Undocumented immigrant Latinas had lower frequency of soda consumption in past week and lower odds of taking blood pressure medication among those with high blood pressure than USborn Latinas. There were no differences for men.

Our study has limitations that should be noted. First, we were limited to the CVD behavioral risk factor measures available in CHIS. While these measures assess behavioral risk factors related to diet, smoking, and exercise, they are not necessarily comprehensive. Second, as in most observational studies that rely on participants to recall behaviors, our measures are subject to recall bias. Third, our documentation status measure is based on participants reporting whether they were foreign-born and having a green card or being naturalized, which may result in some misclassification, particularly for international students and those with temporary visas. Nevertheless, any misclassification would result in more conservative estimates for undocumented immigrants. Finally, our observations are limited to California. While California has the largest undocumented immigrant population compared with other states, our findings may not be entirely generalizable to other parts of the country.

\section{Conclusions}

This study shows that documentation status is important to consider when studying heart disease prevention for Latinos. To the extent that men are more likely to suffer from CVD, and undocumented Latino men have behavioral risk profiles similar to US-born Latinos, we would not expect to see better CVD outcomes for undocumented immigrants than the US-born. The pattern is similar among Latino green card holders and naturalized citizens compared to US-born Latinos, which indicates that there is little or no immigrant health advantage in CVD risk among men, but there likely is among women. Given that undocumented Latino immigrants also have significant barriers to health care, including insurance eligibility, access to health care providers, and language concordant care [9-11], future studies should be cognizant of citizenship and documentation statuses when identifying clinical interventions to reduce CVD outcomes.

\section{Abbreviations \\ ACA: Affordable Care ActANOVA: Analysis of Variance; CHIS: California Health Interview Survey; Cl: Confidence Intervals; CVD: Cardiovascular Disease; FPL: Federal Poverty Level; IRR: Incidence Rate Ratios; OR: Odds Ratio; US: United States}

\section{Acknowledgements}

Not applicable

\section{Authors' contributions}

ANO conceptualized and designed the study, interpreted data results, and drafted all sections of the manuscript. JKP assisted in the literature review, data interpretation, and provided critical feedback on all drafts of the text. BAL assisted in data interpretation and provided edits on all drafts of the manuscript. AVB assisted in data interpretation and provided edits on all drafts of the manuscript. MEDTY assisted in data interpretation and provided critical feedback on all drafts of the manuscript. MLP assisted in providing substantial feedback on all drafts of the manuscript. CKA conducted the literature review, assisted in data interpretation and provided critical feedback on all drafts of the text. SPW oversaw the study design and provided guidance and critical feedback on all drafts of the manuscript. All authors read and approved the final manuscript.

\section{Funding}

Funding for the analyses of the secured CHIS data was provided by a faculty discretionary fund to the corresponding author.

\section{Availability of data and materials}

The data that support the findings of this study are available from the UCLA Center for Health Policy Research, but restrictions apply to the availability of secured data. Public use data are available to the public, and use of secured data is provided by application and permission from the UCLA Center for Health Policy Research. Instructions on how to apply for accessing secured CHIS data can be found here: https://healthpolicy.ucla.edu/chis/data/Pages/ confidential.aspx. 


\section{Ethics approval and consent to participate}

We remotely analyzed secured California Health Interview Survey (CHIS) data through the Data Access Center (DAC) at the UCLA Center for Health Policy Research, which provides a secure, controlled environment that protects the confidentiality of respondents. CHIS has been continuously approved by the UCLA Institutional Review Board to be compliant with human subjects' requirements. CHIS allows for outside researchers to apply for access to their secured data. Applications are reviewed by the CHIS Data Disclosure Review Committee and by the CHIS Principal Investigator. Once an application is approved, programming codes are composed by researchers and emailed to the DAC. Output is then provided to researchers in de-identified form. We received permission from the CHIS Data Disclosure Review Committee and the CHIS Principal Investigator to analyze the secured data. Non-secured CHIS data are publicly available.

\section{Consent for publication}

Not applicable.

\section{Competing interests}

The authors declare that they have no competing interests.

\section{Author details}

'Dornsife School of Public Health, Drexel University, Philadelphia, PA 19104, USA. ${ }^{2}$ Fielding School of Public Health, University of California, Los Angeles, CA 90095, USA. ${ }^{3}$ School of Social Sciences, Humanities and Arts, University of California, Merced, CA 95343, USA.

Received: 13 September 2019 Accepted: 27 April 2020

\section{Published online: 06 May 2020}

\section{References}

1. Heron M. Deaths: Leading causes for 2016. Natl Vital Stat Rep. 2018;67(6).

2. Al Kibria GM. Racial/ethnic disparities in prevalence, treatment, and control of hypertension among US adults following application of the 2017 American College of Cardiology/American Heart Association guideline. Prev Med Rep. 2019;14:100850.

3. Muntner P, Carey RM, Gidding S, Jones DW, Taler SJ, Wright JT, et al. Potential US population impact of the 2017 ACC/AHA high blood pressure guideline. Circulation. 2018;137(2):109-18.

4. Daviglus ML, Pirzada A, Durazo-Arvizu R, Chen J, Allison M, Avilés-Santa L, et al. Prevalence of low cardiovascular risk profile among diverse Hispanic/ Latino adults in the United States by age, sex, and level of acculturation: the Hispanic community health study/study of Latinos. J Am Heart Assoc. 2016; 5(8):e003929.

5. Abraído-Lanza AF, Chao MT, Flórez KR. Do healthy behaviors decline with greater acculturation?: implications for the Latino mortality paradox. Soc Sci Med. 2005;61(6):1243-55.

6. Torres JM, Young MT. A life-course perspective on legal status stratification and health. SSM Popul Health. 2016;2:141-8.

7. Krogstad J, Passel, J., Cohn, D'Vera. 5 Facts about illegal immigration in the U.S. https://www.pewresearch.org/fact-tank/2018/11/28/5-facts-about-illegalimmigration-in-the-u-s/: PEW Research; 2018 [Available from: https://www. pewresearch.org/fact-tank/2018/11/28/5-facts-about-illegal-immigration-inthe-u-s/.

8. Wallace SP, Torres J, Sadegh-Nobari T, Pourat N, Brown ER. Undocumented immigrants and health care reform. UCLA Center Health Policy Res. 2012:1-48.

9. Ortega AN, Fang H, Perez VH, Rizzo JA, Carter-Pokras O, Wallace SP, et al. Health care access, use of services, and experiences among undocumented Mexicans and other Latinos. Arch Intern Med. 2007;167(21):2354-60.

10. Ortega AN, McKenna RM, Kemmick Pintor J, Langellier BA, Roby DH, Pourat $\mathrm{N}$, et al. Health care access and physical and behavioral health among undocumented Latinos in California. Med Care. 2018;56(11):919-26.

11. Vargas Bustamante A, Fang H, Garza J, Carter-Pokras O, Wallace SP, Rizzo JA, et al. Variations in healthcare access and utilization among Mexican immigrants: the role of documentation status. J Immigr Minor Health. 2012; 14(1):146-55.

12. Alcala HE, Albert SL, Roby DH, Beckerman J, Champagne P, Brookmeyer R, et al. Access to care and cardiovascular disease prevention: a cross-sectional study in 2 Latino communities. Medicine (Baltimore). 2015;94(34):e1441.

13. Young MT, Pebley AR. Legal status, time in the USA, and the well-being of Latinos in Los Angeles. J Urban Health. 2017;94(6):764-75.
14. California Health Interview Survey. Design \& Methods | UCLA Center for Health Policy Research UCLA: UCLA Center for Health Policy Research; 2018 [Available from: http://healthpolicy.ucla.edu/chis/design/Pages/overview. aspx.

15. Bustamante AV, McKenna RM, Viana J, Ortega AN, Chen J. Access-to-care differences between Mexican-heritage and other Latinos in California after the affordable care act. Health Aff (Millwood). 2018;37(9):1400-8.

16. Young MT, Madrigal DS. Documenting legal status: a systematic review of measurement of undocumented status in health research. Public Health Rev. 2017;38(1):26.

17. Ortega AN, Horwitz SM, Fang H, Kuo AA, Wallace SP, Inkelas M. Documentation status and parental concerns about development in young US children of Mexican origin. Acad Pediatr. 2009;9(4):278-82.

18. Measurement error in citizenship and immigration status among Mexicanborn respondents in the California Health Interview Survey [press release]. 2017

19. US Department of Health and human Services and US Department of Agriculture. 2015-2020 Dietary Guidelines for Americans. [Available from: https://health.gov/dietaryguidelines/2015/guidelines/2015.

20. Consejo Nacional de Población. Nuevos patrones de la migración MéxicoEstados Unidos: Consejo Nacional de Población; 2010 [Available from: http://www.conapo.gob.mx/work/models/CONAPO/intensidad_migratoria/ pdf/Nuevos_patrones.pdf.

21. Pew Research Center. U.S. unauthorized immigration population estimates: estimated unauthorized immigrant population, by state, 2016. Washington, D.C: Pew Research Center; 2016. [Available from: http://www.pewhispanic. org/interactives/unauthorized-immigrants/.

22. Migration Policy Institute. Profile of the unauthorized population: United States [Available from: https://www.migrationpolicy.org/data/unauthorizedimmigrant-population/state/US.

23. Stern D, Piernas C, Barquera S, Rivera JA, Popkin BM. Caloric beverages were major sources of energy among children and adults in Mexico, 1999-2012. J Nutr. 2014:144(6):949-56.

24. Sanchez-Pimienta TG, Batis C, Lutter CK, Rivera JA. Sugar-sweetened beverages are the main sources of added sugar intake in the Mexican population. J Nutr. 2016;146(9):1888s-96s.

25. Organisation for Economic Co-operation and Development (OECD). Obesity update: Organisation for Economic Co-operation and Development; 2017 [Available from: http://www.oecd.org/health/obesity-update.htm.

26. Colchero MA, Salgado JC, Unar-Munguía M, Molina M, Ng S, RiveraDommarco JA. Changes in prices after an excise tax to sweetened sugar beverages was implemented in Mexico: evidence from urban areas. PLoS One. 2015;10(12):e0144408.

27. Passel J, Cohn, D. As Mexican share declined, US unauthorized immigrant population fell in 2015 below recession level: Pew Research Center; 2017 [Available from: https://www.pewresearch.org/fact-tank/2017/04/25/asmexican-share-declined-u-s-unauthorized-immigrant-population-fell-in-2015below-recession-level/.

28. Hall M. Residential integration on the new frontier: immigrant segregation in established and new destinations. Demography. 2013;50(5):1873-96.

29. Ramirez A. Food \& Latino Kids: A research review: Salud America!; 2016 [Available from: https://salud-america.org/food-latino-kids-research-review/.

30. Langellier BA. Consumption and expenditure on food prepared away from home among Mexican adults in 2006. Salud Publica Mex. 2015;57(1):4-13.

31. Lee $H$, Zakhary BL, Firek MA, Lange $H$, Gomez CA, Firek A. The prevalence and impact of diabetes mellitus among undocumented immigrants in an indigent care program in Riverside, California. Diabetes. 2018;67(Supplement 1):1625.

32. McKenna RM, Alcala HE, Le-Scherban F, Roby DH, Ortega AN. The affordable care act reduces hypertension treatment disparities for Mexican-heritage Latinos. Med Care. 2017;55(7):654-60.

\section{Publisher's Note}

Springer Nature remains neutral with regard to jurisdictional claims in published maps and institutional affiliations. 